\title{
Pulmonary Artery Mapping for Differential Diagnosis of Left-Sided Atrial Tachycardia
}

\author{
Yukoh Hirai, MD; Yukiko Nakano, MD; Hideya Yamamoto, MD; Hiroshi Ogi, MD; \\ Yoshiyuki Yamamoto, MD; Kazuyoshi Suenari, MD; Noboru Oda, MD; Shigeyuki Ueda, MD; \\ Yuko Makita, MD; Kenta Kajihara, MD; Yasuki Kihara, MD
}

\begin{abstract}
Background: Distinguishing left- and right-sided atrial tachycardia (AT) is often challenging. The coronary sinus (CS) provides information only concerning the anterior left atrium (LA). Potentials recorded in the pulmonary artery (PA) have been substituted for those of the upper posterior LA because of their anatomical relationship.
\end{abstract}

Methods and Results: Three patterns were designed, using potentials in the PA, right atrium (RA) and CS, to
predict the side of AT. Two patterns were for left-sided AT and 1 pattern was for right-sided AT. Ten left-sided and
11 right-sided ATs were investigated regardless of mechanism. Electrode catheters were inserted in the RA, His bundle
region, and CS, and an ablation catheter was inserted into the left and/or right PA. The sequences from these cath-
eters were analyzed before detailed electroanatomical mapping. Patterns were obtained for 20 of 21 ATs. The mecha-
nism was focal in 16 ATs and macroreentry in 5 . The method predicted left-sided AT with a sensitivity of $78 \%$, a
specificity of $100 \%$, a positive predictive value of $100 \%$, a negative predictive value of $84 \%$, and an accuracy of $90 \%$.

Conclusions: The use of potentials in PA combined with conventional RA and CS electrograms is useful for distinguishing left-sided AT from right-sided AT, regardless of mechanism. (Circ J 2013; 77: 345-351)

Key Words: Ablation; Atrial tachycardia; Differential diagnosis; Pulmonary artery mapping

$\mathbf{R}$ adiofrequency $(\mathrm{RF})$ catheter ablation is presently the first-line therapy for atrial tachycardia (AT). The majority of ATs originate from the right atrium (RA), and only a few originate from the left atrium (LA). ${ }^{1-3}$ Differentiation between right- and left-sided AT is often challenging. The wavefront of left-sided AT usually propagates into the RA, either anteriorly through the coronary sinus (CS) or posteriorly through Bachman's bundle. The CS catheter, however, which is commonly used for assessing the origin of AT during cardiac electrophysiological studies (EPS), can provide information only about the anterior aspect of the LA. ${ }^{4-7}$

Pulmonary arteries (PAs) are known to provide far-field potentials of the upper site or roof of the LA. ${ }^{89}$ We thus designed 3 sequence patterns using potentials in the PA, RA and CS: 2 for left-sided and one for right-sided ATs. The purpose of this study was to determine whether left-sided AT could be distinguished from right-sided AT using the designed sequence patterns.

\section{Methods}

\section{Patients and AT}

A total of 21 ATs in 20 patients were included in the study (12 men and 8 women; median age, 59 years; range, 34-76 years). The ATs were prospectively classified into the new sequence patterns using PA mapping. Subsequently, we confirmed the origin of the ATs using an electroanatomical mapping system and validated the reliability of the sequence patterns.

The Institutional Ethics Committee of the Graduate School of Biomedical Science, Hiroshima University, approved all procedures. Cardiac EPS was performed in the laboratory after obtaining written informed consent from all subjects.

\section{Catheter Deployment}

A 20-electrode Halo catheter (2-mm interelectrode spacing) was placed in the RA to record electrograms of the posterior septum and lateral aspects of the RA. A multi-electrode catheter was placed in the His bundle region through the right inguinal vein, and a decapolar-electrode catheter (2-mm interelectrode spacing) was introduced into the CS through the right internal jugular vein. When spontaneous AT was not observed, AT was induced by programmed stimulation and/or burst pacing with or without isoproterenol infusion $(0.5-2 \mu \mathrm{g} / \mathrm{min})$. A deflectable ablation catheter (4-mm tip electrode) was inserted into the left PA and/or right PA until the catheter went over the roof of the LA and was curved down as deeply as possible

Received May 30, 2012; revised manuscript received August 28, 2012; accepted September 27, 2012; released online October 26, 2012 Time for primary review: 29 days

Department of Cardiovascular Medicine, Hiroshima University Graduate School of Biomedical Science, Hiroshima, Japan

Mailing address: Yukiko Nakano, MD, Department of Cardiovascular Medicine, Hiroshima University Graduate School of Biomedical Science, 1-2-3 Kasumi, Minami-ku, Hiroshima 734-8551, Japan. E-mail: nakanoy@ hiroshima-u.ac.jp

ISSN-1346-9843 doi:10.1253/circj.CJ-12-0698

All rights are reserved to the Japanese Circulation Society. For permissions, please e-mail: cj@j-circ.or.jp 




Figure 1. (Upper) Fluoroscopic and (Lower) schematic images of catheter placement. Bold arrows, right and left pulmonary arteries. CSd, coronary sinus distal; CSm, coronary sinus middle; CSp, coronary sinus proximal; $\mathrm{FO}$, fossa ovale; HBR, His bundle region; $\mathrm{Hd}$, Halo distal; Hm, Halo middle; Hp, Halo proximal; LA, left atrium; LAO, left anterior oblique; LPA, left pulmonary artery; MA, mitral annulus; $\mathrm{RA}$, right atrium; $\mathrm{RAO}$, right anterior oblique; RPA, right pulmonary artery; SVC, superior vena cava; TA, tricuspid annulus.

in order to avoid picking up the far-field potentials of superior vena cava (SVC) potentials. Fluoroscopy and schematic drawings of the anatomical relationships and catheter deployment are given in Figure 1. Bipolar intracardiac electrograms filtered between 30 and $500 \mathrm{~Hz}$ were recorded and stored digitally on a computerized system using 12-lead surface electrocardiography (ECG).

\section{PA Mapping and Sequence Analysis}

We designed 3 sequence patterns $(\mathbf{A}-\mathbf{C})$ using potentials in the PA, RA and CS. Schematic images are given in Figure 2, and an example of each tracing is shown in Figure 3. Patterns A and $\mathbf{B}$ were for left-sided AT, whereas Pattern $\mathbf{C}$ was for rightsided AT. In Pattern A, potentials in the PA were detected earlier than proximal Halo and His bundle region potentials, and this was irrespective of the CS sequence. In Pattern B, the middle CS was activated before the proximal CS, irrespective of whether or not the potentials in PA were earlier than the other potentials. In Pattern C, the CS sequence had a proximal-to-distal pattern, and potentials in the PA were detected after the proximal Halo and His bundle region potentials, irrespective of the Halo sequence. We assumed that both focal and macroreentrant AT was present in this study. Although the earliest sites do not exist in macroreentrant AT, the wavefront from the LA would propagate to the RA posteriorly or anteriorly. If the wavefront propagates posteriorly, the posterior LA is activated earlier than the posterior RA. If the wavefront propagates anteriorly, the CS sequence has a middle-to-proximal pattern. In the latter case, a distal-to-proximal pattern is not necessary because focal AT from the middle $\mathrm{CS}$ area would show a centrifugal pattern beginning from the middle CS.
AT was classified according to the 3 described sequence patterns before detailed mapping and ablation.

In the first several cases, entrainment pacing in the PA was attempted at the maximum output of $10 \mathrm{~V} / 2 \mathrm{~ms}$. The stimulation, however, never captured any tissue so this procedure was excluded from the protocol.

\section{Electroanatomical Mapping and Ablation}

After pattern classification, we used an electroanatomical mapping system $\left(\mathrm{CARTO}^{\circledR}\right.$; Biosense Webster, Diamond Bar, CA, USA) to map, diagnose, and ablate AT in all patients. A macroreentrant mechanism was diagnosed using CARTO mapping, with a head-to-tail pattern satisfying the tachycardia cycle length. In some cases, we performed entrainment pacing in the lateral RA, the cavotricuspid isthmus, and the proximal and distal CS after CARTO mapping. The postpacing intervals were measured at each location to identify the circuits. ${ }^{3}$ Entrainment pacing, however, often terminates tachycardia; therefore, we did not attempt entrainment pacing in most cases.

Ablation was performed after suspected foci or circuits were identified by mapping. When focal AT was suspected, RF currents were delivered using a non-irrigated catheter with a 4$\mathrm{mm}$ tip under a controlled temperature of $55-60^{\circ} \mathrm{C}$ with a maximum power of $30-50 \mathrm{~W}$ in the earliest activation sites. If the AT was not affected within $10 \mathrm{~s}$, the current was discontinued and detailed mapping was reinitiated until better points were identified. If the RF current application prolonged the cycle length of the AT or terminated the AT within $10 \mathrm{~s}$, the current application was continued for 30-60 s. Successful ablation was defined as non-inducibility of AT. When macroreentrant AT was suspected, linear ablations of the anticipated critical isthmus were performed using the same ablation protocol described 




Pattern A



Pattern B
LPA

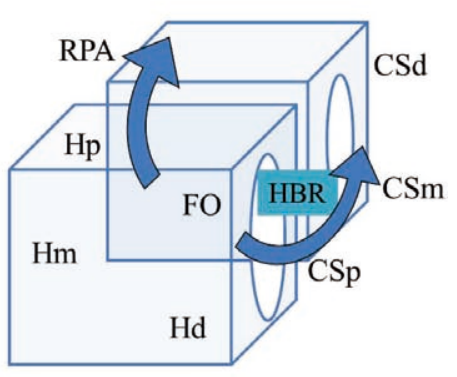

Pattern C

Figure 2. Schematic images of the 3 new sequence patterns. Patterns $\mathbf{A}$ and $\mathbf{B}$ are for left-sided atrial tachycardia (AT) and Pattern C is for right-sided AT. CSd, coronary sinus distal; CSm, coronary sinus middle; CSp, coronary sinus proximal; FO, fossa ovale; HBR, His bundle region; Hd, Halo distal; Hm, Halo middle; Hp, Halo proximal; LPA, left pulmonary artery; RPA, right pulmonary artery.
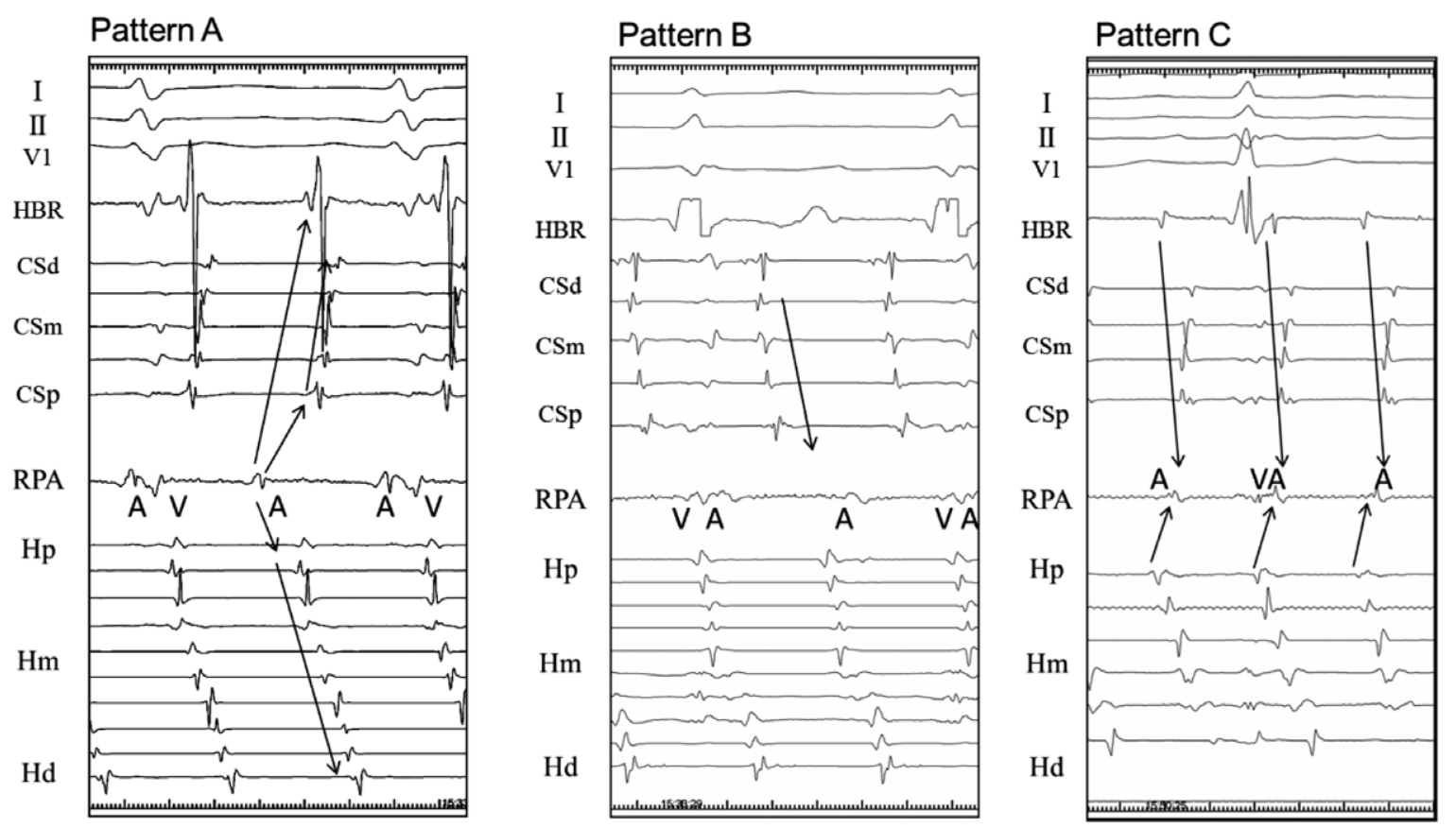

Figure 3. Intracardiac tracings of each pattern. (Pattern A) Potentials in right pulmonary artery (PA) were detected earlier than proximal Halo and His bundle region potentials. (Pattern B) The middle coronary sinus (CS) was activated earlier than the proximal CS. (Pattern C) The proximal Halo and His bundle region were activated earlier than the right PA. CSd, coronary sinus distal; CSm, coronary sinus middle; CSp, coronary sinus proximal; HBR, His bundle region; Hd, Halo distal; Hm, Halo middle; Hp, Halo proximal; RPA, right pulmonary artery.

here until the creation of block lines was confirmed.

\section{Statistical Analysis}

Continuous valuables are presented as mean $\pm \mathrm{SD}$, median and range, or proportions, as appropriate.

\section{Results}

Patient Characteristics

Patient characteristics are listed in Table. Eight patients had underlying heart disease and 6 had a history of open heart surgery; two of the latter had undergone a modified maze procedure, which is a box-like isolation of the posterior LA together 


\begin{tabular}{|c|c|c|c|c|c|c|c|c|c|c|c|c|}
\hline AT & Pattern & Diagnosis & Side & TCL & $\begin{array}{l}\text { Surface } P \\
\text { wave }\end{array}$ & $\begin{array}{c}\text { Entrain- } \\
\text { ment }\end{array}$ & $\begin{array}{l}\text { Mecha- } \\
\text { nism }\end{array}$ & $\begin{array}{c}\text { CS } \\
\text { sequence }\end{array}$ & Patient & Sex & $\begin{array}{c}\text { Age } \\
\text { (years) }\end{array}$ & $\begin{array}{l}\text { Underlying } \\
\text { heart disease }\end{array}$ \\
\hline 1 & A & MA CCW AFI & LA & 277 & $\begin{array}{l}\text { Very low } \\
\text { amplitude }\end{array}$ & Not done & $\begin{array}{l}\text { Macro- } \\
\text { reentry }\end{array}$ & $P$ to $D$ & 1 & M & 76 & None \\
\hline 2 & A & $\begin{array}{l}\text { APCs from } \\
\text { LSPV }\end{array}$ & LA & Irregular & Discernible & Not done & Focal & Equivocal & 2 & M & 45 & None \\
\hline 3 & B & MA CW AFI & LA & 277 & $\begin{array}{l}\text { Very low } \\
\text { amplitude }\end{array}$ & Not done & $\begin{array}{l}\text { Macro- } \\
\text { reentry }\end{array}$ & $D$ to $P$ & 3 & M & 65 & $\begin{array}{l}\text { AVR, MVP, } \\
\text { modified-Maze }\end{array}$ \\
\hline 4 & A & Incisional AT & LA & 240 & Discernible & Not done & $\begin{array}{l}\text { Macro- } \\
\text { reentry }\end{array}$ & Equivocal & 3 & M & 65 & $\begin{array}{l}\text { AVR, MVP, } \\
\text { modified-Maze }\end{array}$ \\
\hline 5 & C & $\begin{array}{l}\text { RIPV antrum } \\
\text { AT }\end{array}$ & LA & 280 & Discernible & $\begin{array}{c}\text { Not } \\
\text { entrainable }\end{array}$ & Focal & $P$ to $D$ & 4 & M & 35 & None \\
\hline 6 & B & $\begin{array}{l}\text { RSPV antrum } \\
\text { AT }\end{array}$ & LA & 283 & $\begin{array}{l}\text { Very low } \\
\text { amplitude }\end{array}$ & $\begin{array}{c}\text { Not } \\
\text { entrainable }\end{array}$ & Focal & $D$ to $P$ & 5 & M & 63 & $\begin{array}{l}\text { HCM, } \\
\text { s/p MVR }\end{array}$ \\
\hline 7 & NA & Incisional AT & LA & 213 & Discernible & $\begin{array}{l}\mathrm{PPI}=\mathrm{CS} \\
\text { ostium }\end{array}$ & $\begin{array}{l}\text { Macro- } \\
\text { reentry }\end{array}$ & $P$ to $D$ & 6 & M & 68 & $\begin{array}{c}\text { DVR, } \\
\text { modified-Maze }\end{array}$ \\
\hline 8 & C & $\begin{array}{l}\text { AT from left } \\
\text { septum }\end{array}$ & LA & 375 & Discernible & Not done & Focal & $P$ to $D$ & 7 & $\mathrm{~F}$ & 43 & $\begin{array}{l}\text { LA myxoma- } \\
\text { ectomy }\end{array}$ \\
\hline 9 & B & $\begin{array}{l}\text { AT from lateral } \\
\text { MA }\end{array}$ & LA & 495 & Discernible & Not done & Focal & $D$ to $P$ & 8 & M & 34 & None \\
\hline 10 & B & $\begin{array}{l}\text { AT from left } \\
\text { appendage }\end{array}$ & LA & 525 & Discernible & Not done & Focal & $D$ to $P$ & 9 & $\mathrm{~F}$ & 45 & ASD closure \\
\hline 11 & C & $\begin{array}{l}\text { Anteroseptum } \\
\text { AT }\end{array}$ & RA & 380 & $\begin{array}{l}\text { Overlapped } \\
\text { on T wave }\end{array}$ & Not done & Focal & $P$ to $D$ & 10 & M & 62 & ARVC \\
\hline 12 & C & $\begin{array}{l}\text { High posterior } \\
\text { septum AT }\end{array}$ & RA & 400 & $\begin{array}{l}\text { Overlapped } \\
\text { on T wave }\end{array}$ & Not done & Focal & $P$ to $D$ & 11 & M & 43 & ASD closure \\
\hline 13 & C & $\begin{array}{l}\text { CCW typical } \\
\text { Afl }\end{array}$ & RA & 320 & Discernible & $\begin{array}{l}\mathrm{PPI}=\mathrm{CS} \\
\text { ostium }\end{array}$ & $\begin{array}{l}\text { Macro- } \\
\text { reentry }\end{array}$ & $P$ to $D$ & 12 & $\mathrm{~F}$ & 59 & None \\
\hline 14 & C & $\begin{array}{l}\text { Anteroseptal } \\
\text { AT }\end{array}$ & RA & 580 & Discernible & Not done & Focal & $P$ to $D$ & 13 & M & 72 & None \\
\hline 15 & C & $\begin{array}{l}\text { Anteroseptal } \\
\text { AT }\end{array}$ & RA & 388 & Discernible & Not done & Focal & $P$ to $D$ & 14 & M & 70 & None \\
\hline 16 & C & AT from CTI & RA & 320 & $\begin{array}{l}\text { Discernible } \\
\text { (ATP used) }\end{array}$ & Not done & Focal & $P$ to $D$ & 15 & $\mathrm{~F}$ & 34 & None \\
\hline 17 & C & PAF from SVC & RA & Irregular & $\begin{array}{l}\text { Overlapped } \\
\text { on T wave }\end{array}$ & Not done & Focal & $P$ to $D$ & 16 & $\mathrm{~F}$ & 42 & None \\
\hline 18 & C & Anterior TA AT & RA & 370 & $\begin{array}{l}\text { Overlapped } \\
\text { on T wave }\end{array}$ & Not done & Focal & $P$ to $D$ & 17 & M & 39 & $\begin{array}{l}\text { Tachycardia } \\
\text { cardiomyopathy }\end{array}$ \\
\hline 19 & C & $\begin{array}{l}\text { High crista } \\
\text { terminalis AT }\end{array}$ & RA & 382 & $\begin{array}{l}\text { Overlapped } \\
\text { on T wave }\end{array}$ & Not done & Focal & $P$ to $D$ & 18 & $\mathrm{~F}$ & 55 & None \\
\hline 20 & C & Para-His AT & RA & 480 & $\begin{array}{l}\text { Overlapped } \\
\text { on T wave }\end{array}$ & Not done & Focal & $P$ to $D$ & 19 & $\mathrm{~F}$ & 75 & None \\
\hline 21 & C & $\begin{array}{l}\text { Posterolateral } \\
\text { AT }\end{array}$ & RA & 470 & Discernible & Not done & Focal & $P$ to $D$ & 20 & $\mathrm{~F}$ & 61 & None \\
\hline
\end{tabular}

$\mathrm{AFI}$, atrial flutter; APC, atrial premature complex; ARVC, arrhythmogenic right ventricular cardiomyopathy; ASD, atrial septal defect; AT, atrial tachycardia; ATP, adenosine triphosphate; AVR, aortic valve replacement; CCW, counterclockwise; CS, coronary sinus; CTI, cavotricuspid isthmus; CW, clockwise; DVR, dual valve replacement (aortic and mitral valves); HCM, hypertrophic cardiomyopathy; LA, left atrium; LSPV, left superior pulmonary vein; MA, mitral annulus; MVP, mitral valvuloplasty; MVR, mitral valve replacement; NA, not applicable; PAF, paroxysmal atrial fibrillation; PPI, post-pacing interval; RA, right atrium; RIPV, right inferior pulmonary vein; RSPV, right superior pulmonary vein; SVC, superior vena cava; TA, tricuspid annulus; TCL, tachycardia cycle length.

with all 4 pulmonary veins (PVs) for atrial fibrillation. ${ }^{10}$ One patient suffered tachycardia cardiomyopathy due to AT.

\section{PA Mapping and Sequence Analysis}

Both right and left PA mapping was performed for 9 ATs, but only right PA mapping was performed for the other ATs. Distinct but low-frequency electrograms, which often needed to be digitally magnified for analysis, were obtained by PA mapping of all ATs. Sequence analyses performed using potentials in PA were applicable to all ATs except for AT 7. We could not classify AT 7 into any pattern because of extensive double potentials, which would represent double potentials of the upper site of the LA due to the incision line of the modified-Maze procedure (Figure 4). Three ATs were classified as Pattern A, 4 as Pattern B, and 13 as Pattern C (Table). PA mapping was easily performed, and no complications were related to this technique.

\section{AT Characteristics and Ablation Outcome}

A total of 21 ATs were studied in 20 patients. Characteristics of the ATs are listed in Table. AT 4 occurred 3 months after successful ablation of AT 3 in the same patient. The mean AT cycle length was $366 \pm 81 \mathrm{~ms}$ (AT 2 and 16 were excluded because of their irregular cycle length). P waves were discernible on a surface ECG in 12 ATs (57.1\%).

Ten left-sided ATs (AT 1-10) and 11 right-sided ATs (AT 

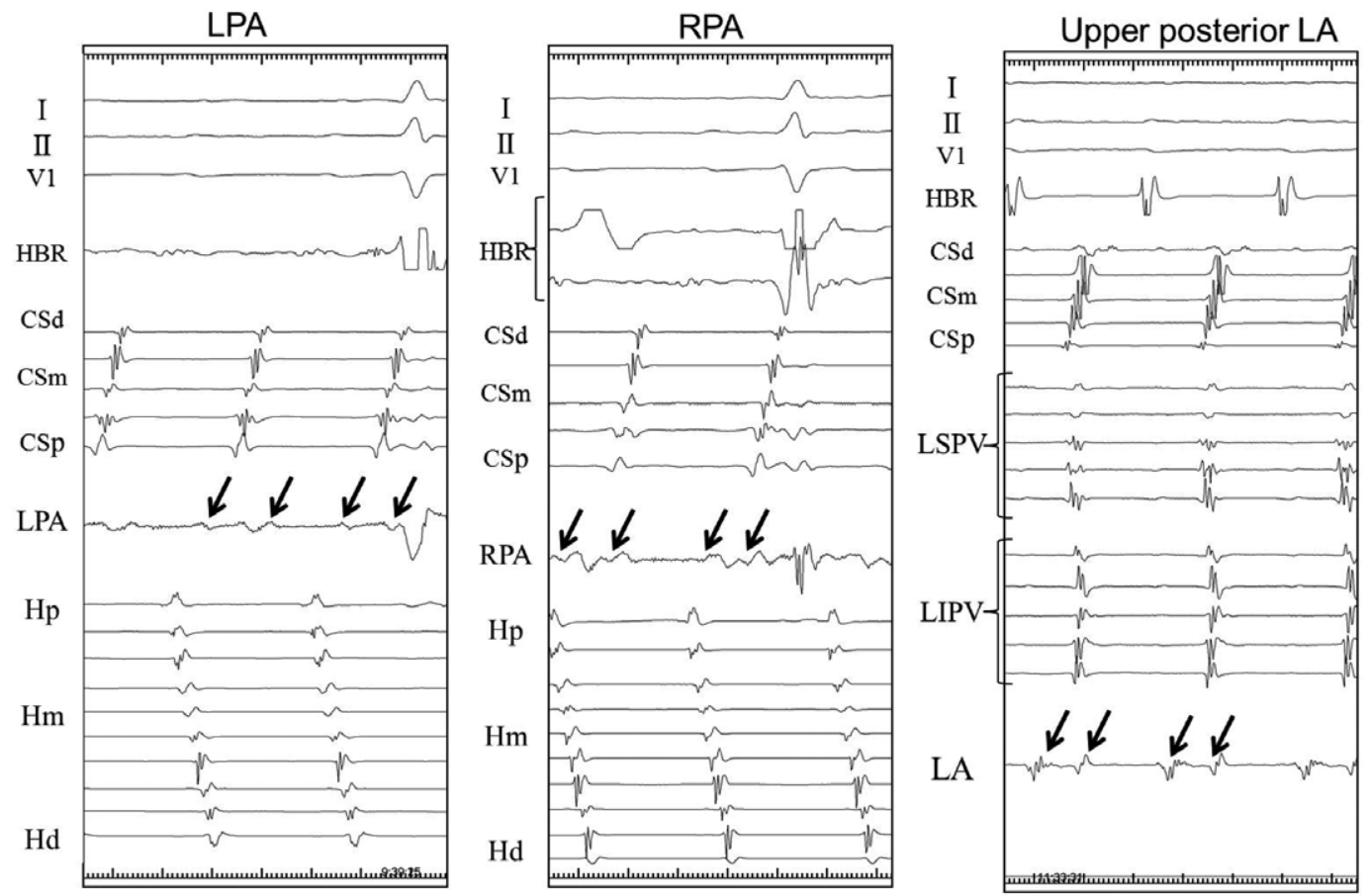

Figure 4. Extensive double potentials in atrial tachycardia (AT) 7 for left pulmonary artery (LPA), right pulmonary artery (RPA), and upper posterior left atrium (upper posterior LA) potentials. These double potentials precluded sequence analysis in this AT. Such double potentials were recorded in PAs and the upper posterior LA, but not in the right atrium (RA). CSd, coronary sinus distal; CSm, coronary sinus middle; CSp, coronary sinus proximal; HBR, His bundle region; Hd, Halo distal; Hm, Halo middle; Hp, Halo proximal; LIPV, left inferior pulmonary vein; LSPV, left superior pulmonary vein.


Figure 5. dots, ablation sites; white dots, successful ablation sites. These CARTO maps clearly demonstrate that the upper posterior areas of the left atrium (LA) were activated later than the right septum in both ATs. LAT, local activation time map; LIPV, left inferior pulmonary vein; LSPV, left superior pulmonary vein; RA, right atrium; RIPV, right inferior pulmonary vein; RSPV, right superior pulmonary vein; SVC, superior vena cava. 
11-21) were diagnosed on CARTO mapping and successful ablation.

Of the 21 ATs, 16 were diagnosed as having a focal mechanism and 5 were diagnosed as having a macroreentrant mechanism. Successful ablation was achieved in all right-sided ATs. Among the 10 left-sided ATs, however, successful ablation was not achieved in AT 4 because of difficulties in terminating the ATs with a non-irrigated ablation catheter.

\section{Reliability of PA Mapping in the Differential Diagnosis of Left- or Right-Sided AT}

All ATs classified as Pattern A or B originated on the left side. Eleven of 13 ATs classified as Pattern $\mathbf{C}$ had a right-sided origin, whereas the remaining 2 ATs (AT 5 and 8) had a leftsided origin. AT 5 originated from the bottom of the right inferior PV antrum, and AT 8 originated in the mid-left septum. CARTO $^{\circledR}$ activation maps of these 2 ATs clearly showed that the upper posterior aspect of the LA was activated later than the RA septal areas in both cases (Figure 5).

Although classification of Pattern B depended only on CS electrograms and did not require PA mapping, fewer than half of the left-sided ATs had this pattern. With CS electrograms alone, only Pattern B (ie, the CS sequence) could predict leftsided AT with a sensitivity of $40 \%$, a specificity of $100 \%$, a positive predictive value (PPV) of $100 \%$, a negative predictive value (NPV) of $63 \%$, and an accuracy of $70 \%$. The present method, in which PA mapping and the designed sequence analyses were added to the CS electrograms, predicted left-sided AT with a sensitivity of $78 \%$, specificity of $100 \%$, PPV of $100 \%$, NPV of $84 \%$, and accuracy of $90 \%$. Therefore, PA mapping improves sensitivity, NPV, and accuracy without any loss of specificity or PPV.

\section{Discussion}

We devised new sequence analyses using PA mapping and CS electrograms to differentially diagnose left-sided AT from rightsided AT. This approach was safe and reliable, regardless of the mechanism of tachycardia.

Although a CS catheter is used for EPS performed to acquire left-sided information, the catheter provides information only about the anterior aspect of the LA. As shown in the present study, approximately half of the left-sided ATs had proximal-to-distal CS sequences; therefore, information obtained using the CS catheter is not sufficient to differentiate AT in the LA from AT in the RA if wavefronts from LA AT are propagated from the posterior LA.

Electrograms recorded in the right and left PAs are known to represent far-field potentials of the upper posterior aspect or the roof of the LA because the PA that runs over the LA diverges into right and left PAs behind the LA and PVs. ${ }^{8,9} \mathrm{We}$ designed 3 new sequence patterns, two of which were for leftsided AT. Pattern A was designed for posterior conduction from the LA to the RA, thus substituting potentials in the PA for posterior LA potentials. In this study, one-third of the leftsided ATs were classified as Pattern A, whereas fewer than half were classified as Pattern B. Although Pattern B conventionally indicates left-sided AT and does not require PA mapping, the present study showed that PA mapping in combination with sequence analysis provided additional value relative to conventional methods. This method also avoids unnecessary performance of detailed RA mapping, trans-septal puncture, and exploratory LA mapping. The present method does not require detailed PA mapping to identify the earliest site in the PAs. We simply advanced an ablation catheter deeply into the PA to locate the distal tip behind the LA, ensuring that this mapping was easy and was not time-consuming.

We propose a stepwise strategy for successful diagnosis. First, if Halo, His bundle region, and CS sequences do not indicate typical atrial flutter, PA mapping should be performed. If the PA and CS patterns indicate left-sided AT, then transseptal LA mapping should be performed because the specificity and PPV are $100 \%$. But if the pattern indicates right-sided AT (ie, Pattern C), detailed RA mapping should be performed. Trans-septal LA mapping should be considered if the earliest activation site is at the septum and unipolar electrograms show an $\mathrm{rS}$ pattern or if there is no distinct focus at the RA septum.

\section{Other Methods}

The $\mathrm{P}$ wave morphologies of focal AT may suggest the foci. ${ }^{1,2,11-16}$ Identification of the morphology, however, is often difficult because $\mathrm{P}$ waves often have low amplitudes and usually overlap the preceding $\mathrm{T}$ waves. $\mathrm{P}$ waves were discernible in 9 of the 16 focal ATs detected in the present study. Although adenosine and adenosine triphosphate are often used to clarify $\mathrm{P}$ waves by inducing a transient atrioventricular (AV) block, AT may be terminated and is sometimes difficult to re-induce. Therefore, the present strategy did not include such drug-related challenges, especially during EPS performed in a laboratory setting.

Double potentials in the posterior RA are reportedly helpful for predicting foci. ${ }^{17,18}$ This technique, however, is useful only for focal AT and requires meticulous posterior RA mapping. In the present study, double potentials were observed in only 8 ATs and those did not always predict the side of their origin (data not shown). Typical placement of a Halo catheter would not always provide useful double potentials.

Yamada et al reported the usefulness of esophageal electrograms for predicting atrial fibrillation foci in the PVs or posterior RA. ${ }^{19}$ Their method may be applicable to focal AT. As for the upper part of the LA, PA potentials can substitute for esophageal potentials, and the present method using PA potentials did not require additional catheters and was non-invasive.

The simple techniques reported by Lee et al and Chang et al appear to be useful for predicting the foci of atrial premature beats and possibly focal AT. ${ }^{20,21}$ If the focal mechanism has been confirmed, these methods can be used in combination with the present method. Catheter placement for PA mapping can provide sufficient information for these methods.

As for macroreentrant AT, entrainment mapping was useful for localization. In the present study, the majority of ATs had a focal mechanism. One difficulty with entrainment pacing is that it often terminates AT, and sometimes, this is very difficult to re-induce in the laboratory.

\section{Clinical Implications}

This study included 4 patients with a history of open heart surgery within the LA. As demonstrated here, even for a patient with a history of box-like PV isolation, PA mapping provides far-field potentials of excitable LA regions, which can aid in diagnosis. Ablation for atrial fibrillation has been widely performed; therefore, new AT that emerges after the procedure is becoming an additional problem. ${ }^{22,23}$ PA mapping may provide useful information in these cases.

\section{Study Limitations}

This study had a number of limitations. First, the number of cases and variety of AT studied was limited. Second, we did not determine whether this technique would be applicable to 
AV reentry tachycardia (AVRT) and AV nodal reentry tachycardia (AVNRT) because these tachycardias were excluded from the study. PA mapping analysis, however, can be applicable in such cases because AVNRT shows centrifugal propagation from the AV nodal area and can be classified as Pattern C, whereas AVRT utilizes the left-sided accessory pathway and can be classified as Pattern B. Third, because the right PA includes far-field potentials of the SVC as well as those of the LA, SVC tachycardia might be misclassified due to mistakenly picking up SVC potentials instead of LA potentials. Although we tried to insert the mapping (ablation) catheter deeply to locate the distal tip behind the LA and successfully classified AT 17 (SVC tachycardia) as right-sided AT using the present method, the special multi-electrode catheter, which can record both SVC and CS potentials, should have been used to avoid this error.

Finally, PA mapping is ineffective for the differential diagnosis of septal tachycardia and lesions in the lower part of the right inferior RA, which may propagate through the septum. PA mapping, however, remains the first choice of diagnostic method because such AT is rare..$^{1,24,25}$

\section{Conclusion}

Sequence analysis using PA potentials in addition to conventional RA and CS potentials is useful for distinguishing leftsided AT from right-sided AT, irrespective of their mechanism.

\section{Acknowledgments}

We thank Dr. Chun-Chieh Wang and Professor Delon Wu for demonstrating that PAs can be mapped. We also thank Enago (www.enago.jp) for assistance with the manuscript.

\section{Disclosures}

None of the authors have any received any financial support.

\section{References}

1. Kistler PM, Roberts-Thomson KC, Haqqani HM, Fynn SP, Singarayar S, Vohra JK, et al. P-wave morphology in focal atrial tachycardia: Development of an algorithm to predict the anatomic site of origin. J Am Coll Cardiol 2006; 48: 1010-1017.

2. Tang CW, Scheinman MM, Van Hare GF, Epstein LM, Fitzpatrick $\mathrm{AP}$, Lee RJ, et al. Use of $\mathrm{P}$ wave configuration during atrial tachycardia to predict site of origin. J Am Coll Cardiol 1995; 26: 13151324.

3. Miyazaki H, Stevenson WG, Stephenson K, Soejima K, Epstein LM. Entrainment mapping for rapid distinction of left and right atrial tachycardias. Heart Rhythm 2006; 3: 516-523.

4. Antz M, Otomo K, Arruda M, Scherlag BJ, Pitha J, Tondo C, et al. Electrical conduction between the right atrium and the left atrium via the musculature of the coronary sinus. Circulation 1998; 98: 17901795.

5. Ho SY, Anderson RH, Sánchez-Quintana D. Atrial structure and fibers: Morphologic bases of atrial conduction. Cardiovasc Res 2002; 54: 325-336.

6. Hoffmann E, Reithmann C, Nimmermann P, Elser F, Dorwarth U, Remp T, et al. Clinical experience with electroanatomic mapping of ectopic atrial tachycardia. Pacing Clin Electrophysiol 2002; 25: $49-56$.

7. Dong J, Zrenner B, Schreieck J, Deisenhofer I, Karch M, Schneider M, et al. Catheter ablation of left atrial focal tachycardia guided by electroanatomic mapping and new insights into interatrial electrical conduction. Heart Rhythm 2005; 2: 578-591.
8. Breithardt G, Seipel L. Recording of left atrial potentials from pulmonary artery in man. Br Heart J 1980; 43: 689-694.

9. Cosio FG, Martin-Penato A, Pastor A, Nunez A, Montero MA, Cantale $\mathrm{CP}$, et al. Atrial activation mapping in sinus rhythm in the clinical electrophysiology laboratory: Observations during Bachmann's bundle block. J Cardiovasc Electrophysiol 2004; 15: 524-531.

10. Sueda T, Nagata H, Orihashi K, Morita S, Okada K, Sueshiro M, et al. Efficacy of a simple left atrial procedure for chronic atrial fibrillation in mitral valve operations. Ann Thorac Surg 1997; 63: 1070-1075.

11. Kistler PM, Fynn SP, Haqqani H, Stevenson IH, Vohra JK, Morton $\mathrm{JB}$, et al. Focal atrial tachycardia from the ostium of the coronary sinus: Electrocardiographic and electrophysiological characterization and radiofrequency ablation. J Am Coll Cardiol 2005; 45: 14881493.

12. Wang YL, Li XB, Quan X, Ma JX, Zhang P, Xu Y, et al. Focal atrial tachycardia originating from the left atrial appendage: Electrocardiographic and electrophysiologic characterization and long-term outcomes of radiofrequency ablation. J Cardiovasc Electrophysiol 2007; 18: $459-464$.

13. Yamada T, Murakami Y, Yoshida Y, Okada T, Yoshida N, Toyama J, et al. Electrophysiologic and electrocardiographic characteristics and radiofrequency catheter ablation of focal atrial tachycardia originating from the left atrial appendage. Heart Rhythm 2007; 4: 12841291.

14. Roberts-Thomson KC, Kistler PM, Haqqani HM, McGavigan AD, Hillock RJ, Stevenson IH, et al. Focal atrial tachycardias arising from the right atrial appendage: Electrocardiographic and electrophysiologic characteristics and radiofrequency ablation. J Cardiovasc Electrophysiol 2007; 18: 367-372.

15. Kistler PM, Sanders P, Hussin A, Morton JB, Vohra JK, Sparks PB, et al. Focal atrial tachycardia arising from the mitral annulus: Electrocardiographic and electrophysiologic characterization. J Am Coll Cardiol 2003; 41: 2212-2219.

16. Chen CC, Tai CT, Chiang CE, Yu WC, Lee SH, Chen YJ, et al. Atrial tachycardias originating from the atrial septum: Electrophysiologic characteristics and radiofrequency ablation. J Cardiovasc Electrophysiol 2000; 11: 744-749.

17. Soejima K, Stevenson WG, Delacretaz E, Brunckhorst CB, Maisel WH, Friedman PL. Identification of left atrial origin of ectopic tachycardia during right atrial mapping: Analysis of double potentials at the posteromedial right atrium. J Cardiovasc Electrophysiol 2000; 11: $975-980$.

18. Yamada T, Murakami Y, Muto M, Okada T, Okamoto M, Toyama J, et al. Electrophysiologic characteristics of atrial tachycardia originating from the right pulmonary veins or posterior right atrium: Double potentials obtained from the posterior wall of the right atrium can be useful to predict foci of atrial tachycardia in right pulmonary veins or posterior right atrium. J Cardiovasc Electrophysiol 2004; 15: $745-751$.

19. Yamada T, Murakami Y, Muto M, Okada T, Okamoto M, Shimizu T, et al. Simple and accurate catheter mapping technique to predict atrial fibrillation foci in the pulmonary veins or posterior right atrium. Heart Rhythm 2004; 1: 427-434.

20. Lee SH, Tai CT, Lin WS, Tsai CF, Hsieh MH, Yu WC, et al. Predicting the arrhythmogenic foci of atrial fibrillation before atrial transseptal procedure: Implication for catheter ablation. J Cardiovasc Electrophysiol 2000; 11: 750-757.

21. Chang KC, Chen JY, Lin YC, Huang SK. Usefulness of interatrial conduction time to distinguish between focal atrial tachyarrhythmias originating from the superior vena cava and the right superior pulmonary vein. J Cardiovasc Electrophysiol 2008; 19: 1231 - 1235.

22. Kumagai K. Catheter ablation of atrial fibrillation: State of the art. Circ J 2011; 75: 2305-2311.

23. Satomi K. Electrophysiological characteristics of atrial tachycardia after pulmonary vein isolation of atrial fibrillation. Circ J 2011; 74: $1051-1058$.

24. Marrouche NF, SippensGroenewegen A, Yang Y, Dibs S, Scheinman MM. Clinical and electrophysiologic characteristics of left septal atrial tachycardia. J Am Coll Cardiol 2002; 40: 1133-1139.

25. Frey B, Kreiner G, Gwechenberger M, Gössinger HD. Ablation of atrial tachycardia originating from the vicinity of the atrioventricular node: Significance of mapping both sides of the interatrial septum. $J$ Am Coll Cardiol 2001; 38: 394-400. 